PROCEEDINGS OF THE

AMERICAN MATHEMATICAL SOCIETY

Volume 123, Number 7, July 1995

\title{
THE ERROR TERM OF HOLOMORPHIC MAPPINGS IN NEVANLINNA THEORY
}

\author{
ZHUAN YE \\ (Communicated by Eric Bedford)
}

\begin{abstract}
We construct a holomorphic mapping from $\mathbb{C}^{m}$ to $\mathbb{P}^{n}$ for any $m$ and $n$ with $m \geq n \geq 1$ which shows Cherry-Lang-Wong's upper bound of the error term of the Second Main Theorem in Nevanlinna theory is essentially the best possible. Thus a question of Serge Lang is answered affirmatively in higher dimensions.
\end{abstract}

\section{INTRODUCTION}

The classical one-dimensional Nevanlinna theory studies the number of solutions to the equation $f(z)=a$ in the disc of radius $r$ as $r$ tends to infinity asymptotically. In the higher-dimensional Nevanlinna theory one studies holomorphic mappings from $\mathbb{C}^{m}$ into an $n$-dimensional complex manifold $X$. One is interested not only in the preimages of points but also in the inverse images of complex analytic subsets of $X$ of positive dimension. Of all the various theorems in Nevanlinna theory, the Second Main Theorem is the most important.

In recent years, there has been considerable interest in finding the precise error term of the Second Main Theorem. Such a question was raised by Serge Lange [4] who was inspired by Vojta's [9] analogy between Nevanlinna theory and Diophantine approximation. In 1990, S. Lang [5] found the best nature of the upper bound of the Second Main Theorem by improving Wong's method in [10] in the equidimensional case. Later, Z. Ye [14] showed all upper bounds of the error terms in $\mathbb{C}$ conjectured by Lang are sharp. Recently, based on Lang's method, Cherry [1] obtained an upper bound for the error term when the dimension of the domain is not less than that of the image space under the assumption that $f$ is a non-degenerate holomorphic mapping. On the other hand, Wong and Stoll [11] also obtained an upper bound for the error term when the dimension of the domain is less than that of the image space under the assumption that $f$ is a linearly non-degenerate meromorphic mapping. However their upper bound has not been verified to be sharp yet. In this paper, we show the main term in the upper bound in Cherry-Lang-Wong's theorem is sharp when the dimension of the domain is not less than the dimension of the image space.

Received by the editors November 1, 1993.

1991 Mathematics Subject Classification. Primary 32H30. 


\section{Preliminaries AND Results}

Let $X$ be a projective manifold of complex dimension $n$ and $D$ be a divisor on $X$. A divisor $D=\sum_{j} D_{j}$ has simple normal crossings if each $D_{j}$ is irreducible, non-singular, and at each point of $X$ there exist complex coordinates $z_{1}, \ldots, z_{n}$ such that $D$ in a neighborhood of this point is defined by $z_{1} \cdots z_{k}=0$ with $k \leq n$. The maximal value of $k$ which can occur is called the complexity of $D$. Let

$$
f: \mathbb{C}^{m} \rightarrow X
$$

be a non-degenerate (i.e., $f\left(\mathbb{C}^{m}\right)$ contains a non-empty open set in $X$ ) holomorphic map and $m \geq n \geq 1$. We always assume in this paper that:

$D=\sum D_{j}$ is an ample divisor and has at worst simple normal crossings of complexity $k(\leq n)$.

$L_{j}=L_{D_{j}}$ is a line bundle associated with $D_{j}$, with a metric $\rho_{j}$.

$s_{j}$ is a holomorphic section of $L_{j}$ such that $\left(s_{j}\right)=D_{j}$ and $\left|s_{j} \circ f\right|_{j}=\left|s_{j} \circ f\right|_{\rho_{j}}$.

$\Omega$ is a volume form on $X$, defining a metric $\kappa$ on the canonical bundle $L_{K}$; so the first Chern form $c_{1}(\kappa)=\operatorname{Ric} \Omega$.

$\gamma_{f}$ is a function with $f^{*} \Omega \wedge \prod_{i=n+1}^{m} \sqrt{-1} / 2 \pi d z_{i} \wedge d \bar{z}_{i}=\gamma_{f} \Psi$, where $\Psi$ is Euclidean volume form in $\mathbb{C}^{m}$.

$\eta$ is a closed and positive $(1,1)$-form such that $c_{1}\left(\rho_{j}\right) \leq \eta$ for all $j$ and $\Omega \leq \eta^{n} / n !$.

Let $\phi(\geq 1)$ and $\psi$ be positive and increasing functions such that

$$
\int_{1}^{\infty} \frac{d u}{u \psi(u)}=b_{0}(\psi)<\infty \text { and } \int_{1}^{\infty} \frac{d x}{\phi(x)}=\infty
$$

In what follows all notations and terms are defined as in [5] unless indicated otherwise, e.g., $T_{f, \eta}, N_{f, D}, N_{f, \text { Ram }}$, etc.

Under the above assumptions, Cherry-Lang-Wong [1], Theorem 11, proved that, when $m \geq n$,

$$
\begin{aligned}
& T_{f, \kappa}(r)+\sum T_{f, \rho_{j}}(r)-N_{f, D}(r)+N_{f, \operatorname{Ram}}(r) \\
& \quad \leq \frac{n}{2} S\left(B T_{f, \eta}^{1+k / n}, b_{1}, \psi, r\right)-\frac{1}{2} \log \gamma_{f}(0)+1,
\end{aligned}
$$

where $S(F, c, \psi, r)=\log F(r)+\log \psi(F(r))+\log \psi(c r F(r) \psi(F(r)))$. Thus when the complexity of a divisor $D$ is $n,(2)$ can be written as

(3) $T_{f, \kappa}(r)+\sum T_{f, \rho_{j}}(r)-N_{f, D}(r)+n_{f, \operatorname{Ram}}(r) \leq n \log T_{f, \eta}(r)+o\left(\log T_{f, \eta}(r)\right)$

for all large $r$ outside a set of finite Lebesgue measure. In this paper we simplify the upper bound of (2), explain the connection between the upper bound and the size of the exceptional set, and show that (3) is sharp. Thus $(n+\varepsilon) \log T_{f}(r)$ is essentially the best possible error term, and we have answered a question of Lang [5] when $m=n$.

Theorem 1. Let $f, D, \rho_{j}, \kappa, \psi, \phi$, and $\eta$ be as above. Suppose that $f(0) \notin D$ and $0 \notin \operatorname{Ram} f$; then

$$
\begin{aligned}
& T_{f, \kappa}(r)+\sum T_{f, \rho_{j}}(r)-N_{f, D}(r)+N_{f, \operatorname{Ram}}(r) \\
& \quad \leq \frac{n+k}{2} \log T_{f, \eta}(r)+n \log \psi\left(T_{f, \eta}(r)\right)+\frac{n}{2} \log \psi(r)-n \log \phi(r)
\end{aligned}
$$

for all $r \geq r_{0}$ outside a set $E$ with $\int_{E} d r / \phi(r)<\infty$. 
Theorem 2. For any integers $m$ and $n$ with $m \geq n \geq 1$, there are a holomorphic mapping from $\mathbb{C}^{m}$ to $\mathbb{P}^{n}$, a divisor $D=\sum_{j=1}^{\bar{q}} D_{j} \subset|L|$, where the $L$ is the hyperplane bundle, and a closed positive $(1,1)$-form $\eta$ such that, for all large $r$,

$$
T_{f, \kappa}(r)+\sum T_{f, \rho_{j}}(r)-N_{f, D}(r)+N_{f, \operatorname{Ram}}(r)=n \log T_{f, \eta}(r)+o\left(\log T_{f, \eta}(r)\right) .
$$

When $\phi(r) \equiv 1$, then the exceptional set in Theorem 1 has finite Lebesgue measure, so (2) is a special case of (4) and (4) is a simplified version of (2). When $\phi(r)=r$, then the exceptional set in Theorem 1 has finite logarithmic measure. However, on the right side of inequality (4) there is an extra term, $-n \log \phi(r)$, which plays an important role when $T_{f, \eta}(r)$ is of finite order (i.e. $T_{f, \eta}(r)$ is approximately equal to $\left.r^{k}\right)$. A more detailed discussion will be found in [13]. In the one-dimensional case, A. Hinkkanen [3] showed the term $\log \psi$ can be completely ignored. Other questions of Lang in [5] were investigated by the author and others in the one-dimensional case (e.g. [12], [8] and [2]).

The function in Theorem 2 is of infinite order (i.e. $\lim \sup \left(\log T_{f, \eta}(r) / \log r\right)$ $=\infty)$ and has a big ramification term. We refer the readers to [13] for the case of finite order maps.

\section{LEMMAS}

Lemma 1. Let $F$ be a positive and increasing function defined for $r>0$ with piecewise continuous derivative. Suppose there exists $r_{1}$ such that $F\left(r_{1}\right) \geq 1$, say. Let $\phi>1$ and $\psi$ be as (1). Then we have the inequality

$$
F^{\prime}(r) \leq F(r) \psi(F(r)) / \phi(r)
$$

for all $r \geq r_{1}$ outside a set $E$ with $\int_{E} d r / \phi(r) \leq b_{0}(\psi)<\infty$.

Proof. Set $E=\left\{r \in\left(r_{1}, \infty\right) ; F^{\prime}(r)>F(r) \psi(F(r)) / \phi(r)\right\}$. Then

$$
\int_{E} \frac{d r}{\phi(r)} \leq \int_{E} \frac{F^{\prime}(r)}{F(r) \psi(F(r))} d r \leq \int_{1}^{\infty} \frac{d u}{u \psi(u)}=b_{0}(\psi) .
$$

Thus the lemma is proved.

Lemma 2. Let $F$ be a positive and increasing function defined for $r>0$ such that the first derivative exists and $F^{\prime}$ is of piecewise continuous derivative. Suppose that both $F(r)$ and $r^{2 m-1} F^{\prime}(r)$ are positive and increasing functions of $r$, and that there exists $r_{1}$ such that $F\left(r_{1}\right) \geq 1$ for $r \geq r_{1}$. Let $b_{1} \geq 1$ be the smallest number such that

$$
b_{1} r^{2 m-1} F^{\prime}(r) \geq 1, \quad \text { for all } r \geq 1 \text {. }
$$

Let $\psi$ and $\phi$ be the same as in Lemma 1. Then, for any $\varepsilon>0$,

$$
\frac{1}{r^{2 m-1}} \frac{d}{d r}\left(r^{2 m-1} \frac{d F}{d r}(r)\right) \leq \varepsilon F(r) \psi^{2}\left(\varepsilon F^{1 / 2}(r)\right) \psi(r) / \phi^{2}(r)
$$

for all $r \geq r_{0}$ outside a set $E$ with $\int_{E} d r / \phi(r)<\infty$.

Proof. For any $\varepsilon>0$, we have from ([14], Lemma 1) that there is a positive and increasing function $\psi_{1}$ such that $\int_{1}^{\infty} d r /\left(r \psi_{1}(r)\right)=b_{0}\left(\psi_{1}\right)<\infty, \psi_{1}(r) \leq$ 
$r^{1 / 2}$, and $\psi_{1}\left(r^{4 m+6}\right) \leq \varepsilon \psi(\varepsilon r)$ for all $r \geq r_{3}$. Applying Lemma 1 to $\psi_{1}$ and $r^{2 m-1} F^{\prime}(r)$ and $\psi$ and $F$ gives

$$
\frac{d}{d r}\left(r^{2 m-1} \frac{d F}{d r}(r)\right) \leq r^{2 m-1} F^{\prime}(r) \psi_{1}\left(r^{2 m-1} F^{\prime}(r)\right) / \phi(r)
$$

for $r \geq r_{1}$ and $r \notin E_{1}$ with $\int_{E_{1}} d r / \phi(r)<\infty$ and

$$
F^{\prime}(r) \leq F(r) \psi_{1}(F(r)) / \phi(r)
$$

for $r \geq 2$ and $r \notin E_{2}$ with $\int_{E_{2}} d r / \phi(r)<\infty$. Thus, as $r \notin E_{1} \cup E_{2}$ and $r \geq r_{0}=\max \left\{r_{1}, r_{2}, r_{3}\right\}$, we obtain that

$$
\begin{aligned}
\frac{1}{r^{2 m-1}} \frac{d}{d r}\left(r^{2 m-1} \frac{d F}{d r}\right) & \leq F(r) \psi_{1}(F(r)) \psi_{1}\left(r^{2 m-1} F(r) \psi_{1}(F(r)) / \phi(r)\right) / \phi^{2}(r) \\
& \leq \varepsilon F(r) \psi\left(\varepsilon F^{1 / 2}(r)\right) \psi\left(\varepsilon F^{1 / 2}(r)\right) \psi(r) / \phi^{2}(r)
\end{aligned}
$$

which implies Lemma 2.

Lemma 3. There is a one-variable entire function $E$ with

$$
\frac{1}{2 \pi} \int_{0}^{2 \pi} \log \left|E\left(r e^{i \theta}\right)\right| d \theta=(1+o(1))(\log r)^{2} \quad(r \rightarrow \infty)
$$

and

$$
\frac{1}{2 \pi} \int_{0}^{2 \pi} \log \left|E^{\prime}\left(r e^{i \theta}\right)\right| d \theta=(1+o(1))(\log r)^{2} \quad(r \rightarrow \infty) .
$$

Proof. Let $r_{n}=\exp ((n(n+1) / 4))$ for $n=1,2,3, \ldots$ and set

$$
E(z)=\prod_{n=1}^{\infty}\left(1+\left(\frac{z}{r_{n}}\right)^{n}\right)
$$

For $r \in\left[r_{k}, r_{k+1}\right)$,

$$
n(E, 0, r)=k(k+1) / 2=2 \log r_{k}=(2+o(1)) \log r \quad(r \rightarrow \infty) .
$$

Consequently,

$$
N(E, 0, r)=(1+o(1))(\log r)^{2} \quad(r \rightarrow \infty) .
$$

Moreover, for $|z|=r \in\left[r_{k}, r_{k+1}\right)$,

$$
\begin{aligned}
\log |E(z)| & =\sum_{n=1}^{k} n \log \left|\frac{z}{r_{n}}\right|+\sum_{n=1}^{k} \log \left|\left(\frac{r_{n}}{z}\right)^{n}+1\right|+\sum_{n=k+1}^{\infty} \log \left|1+\left(\frac{z}{r_{n}}\right)^{n}\right| \\
& =N(E, 0, r)+I_{1}+I_{2} .
\end{aligned}
$$

A straightforward argument shows that $E$ is an entire function and that

$$
\left|I_{1}\right| \leq k \log 2 \leq O(\sqrt{\log r}) \quad(r \rightarrow \infty)
$$

and

$$
\left|I_{2}\right| \leq \log 2+\sum_{n=k+2}^{\infty}\left(\frac{r_{k+1}}{r_{k+2}}\right)^{n} \leq O(1) \quad(r \rightarrow \infty)
$$


It follows from (7), (10), (11), and (12) that

$$
\begin{aligned}
N(E, 0, r) & \leq T(E, r) \leq \log M(E, r) \\
& =\log E(r) \leq(1+o(1)) N(E, 0, r) \quad(r \rightarrow \infty)
\end{aligned}
$$

and so, as $r \rightarrow \infty$,

(14)

$$
m(E, r)=T(E, r)=(1+o(1)) N(E, 0, r) \text { and } m(E, 0, r)=o(T(E, r)) \text {. }
$$

Putting together (14), (13), and (9) we have proved (5). Furthermore, for $r \in$ $\left[r_{k}, r_{k+1}\right)$,

$$
\begin{aligned}
\frac{z E^{\prime}(z)}{E(z)}= & \sum_{n=1}^{k} n+\sum_{n=1}^{k-1} \frac{-n}{1+\left(z / r_{n}\right)^{n}}+\frac{-k}{1+\left(z / r_{k}\right)^{k}} \\
& +\frac{(k+1)\left(z / r_{k+1}\right)^{k+1}}{1+\left(z / r_{k+1}\right)^{k+1}}+\sum_{n=k+2}^{\infty} \frac{n\left(z / r_{n}\right)^{n}}{1+\left(z / r_{n}\right)^{n}} \\
= & n(E, 0, r)+J_{1}+\frac{-k}{1+\left(z / r_{k}\right)^{k}}+\frac{(k+1)\left(z / r_{k+1}\right)^{k+1}}{1+\left(z / r_{k+1}\right)^{k+1}}+J_{2} .
\end{aligned}
$$

For $1 \leq n \leq k-1$ and $r \in\left[r_{k}, r_{k+1}\right)$, we have from the definition of $r_{k}$ that

$$
\left|\frac{z}{r_{n}}\right|^{n} \geq\left|\frac{r_{k}}{r_{k-1}}\right|^{n}=\exp \left(\frac{n(k+2)}{2}\right) \geq e^{n} \geq 1+\frac{n^{3}}{6} .
$$

Thus we obtain from the definition of $J_{1}$ in (15) and (16) that

$$
\left|J_{1}\right| \leq \sum_{n=1}^{k-1} \frac{n}{\left(r / r_{n}\right)^{n}-1} \leq \sum_{n=1}^{k-1} \frac{6 n}{n^{3}}=O(1) \quad(r \rightarrow \infty) .
$$

For $n \geq k+2$ and $r \in\left[r_{k}, r_{k+1}\right)$, similar to above, we get

$$
\left|r_{n} / z\right|^{n} \geq\left(r_{k+2} / r_{k+1}\right)^{n} \geq e^{n}=1+n^{3} / 6
$$

It follows that

$$
\left|J_{2}\right| \leq \sum_{n=k+2}^{\infty} \frac{n}{\left(r_{n} / r\right)^{n}-1} \leq \sum_{n=k+2}^{\infty} \frac{6 n}{n^{3}}=O(1) \quad(r \rightarrow \infty) .
$$

Moreover, if $|a| \geq 1$ and $a \neq-1$, then $\operatorname{Re}\{1 /(1+a)\} \leq 1 / 2$; so, for $r \in\left[r_{k}, r_{k+1}\right)$,

$$
\operatorname{Re}\left\{1 /\left(1+\left(z / r_{k}\right)^{k}\right)\right\} \leq 1 / 2, \quad \text { if }\left(z / r_{k}\right)^{k} \neq-1 .
$$

To estimate the remaining term in (15), we set $T_{k}=\left(r_{k}+r_{k+1}\right) / 2$. Then, for $r \in\left[r_{k}, T_{k}\right]$,

$$
\begin{aligned}
\left(\frac{r_{k+1}}{r}\right)^{k+1} & \geq\left(\frac{2 r_{k+1}}{r_{k}+r_{k+1}}\right)^{k+1}=\left(1+\frac{r_{k+1}-r_{k}}{r_{k+1}+r_{k}}\right)^{k+1} \\
& \geq 1+(k+1)\left(\frac{1}{2}-\frac{r_{k}}{2 r_{k+1}}\right) \geq 1+\frac{1}{2}\left(1-e^{-1 / 2}\right)(k+1)
\end{aligned}
$$


for all large $k$. It follows form (15)-(20), $k=O(\sqrt{\log r})$, and (9) that, for $r \in\left[r_{k}, T_{k}\right]$, and $\left(z / r_{k}\right)^{k} \neq-1$,

$$
\begin{aligned}
\left|\frac{z E^{\prime}(z)}{E(z)}\right| & \geq \operatorname{Re}\left\{\frac{z E^{\prime}(z)}{E(z)}\right\} \\
& \geq n(f, 0, r)-\left|J_{1}\right|-k \operatorname{Re} \frac{1}{1+\left(z / r_{k}\right)^{k}}-\left|\frac{(k+1)\left(z / r_{k+1}\right)^{k+1}}{1+\left(z / r_{k+1}\right)^{k+1}}\right|-\left|J_{2}\right| \\
& \geq n(E, 0, r)+O(1)-\frac{k}{2}-\frac{k+1}{\left|r_{k+1} / z\right|^{k+1}-1}+O(1) \\
& \geq(1+o(1)) n(E, 0, r) \quad(r \rightarrow \infty) .
\end{aligned}
$$

Therefore we have from (21) and (9) that, for $r \in\left[r_{k}, T_{k}\right]$,

$$
\begin{aligned}
\frac{1}{2 \pi} \int_{0}^{2 \pi} \log \left|\frac{E^{\prime}}{E}\left(r e^{i \theta}\right)\right| d \theta & \geq \log n(E, 0, r)-\log r+O(1) \\
& =(-1+o(1)) \log r \quad(r \rightarrow \infty) .
\end{aligned}
$$

To accomplish inequality (22) for $r \in\left[T_{k}, r_{k+1}\right.$ ), we apply Jensen's formula to $E^{\prime} / E$. Then the fact $N(E, 0, r)=N\left(E, 0, T_{k}\right)$ and (22) give that, for all large $k$,

$$
\begin{aligned}
\frac{1}{2 \pi} & \int_{0}^{2 \pi} \log \left|\frac{E^{\prime}}{E}\left(r e^{i \theta}\right)\right| d \theta \\
& =N\left(E^{\prime} / E, 0, r\right)-N\left(E^{\prime} / E, \infty, r\right)+O(1) \\
& =N\left(E^{\prime}, 0, r\right)-N(E, 0, r)+O(1) \\
& \geq N\left(E^{\prime}, 0, T_{k}\right)-N\left(E, 0, T_{k}\right)+O(1) \\
& \geq N\left(E^{\prime} / E, 0, T_{k}\right)-N\left(E^{\prime} / E, \infty, T_{k}\right)+O(1) \\
& =\frac{1}{2 \pi} \int_{0}^{2 \pi} \log \left|\frac{E^{\prime}}{E}\left(T_{k} e^{i \theta}\right)\right| d \theta+O(1) \geq(-1+o(1)) \log T_{k} \\
& \geq(-1+o(1)) \log r \quad(r \rightarrow \infty) .
\end{aligned}
$$

Combining (22), (23), and (5), we see

$$
\begin{aligned}
\frac{1}{2 \pi} \int_{0}^{2 \pi} \log \left|E^{\prime}\left(r e^{i \theta}\right)\right| d \theta & \geq \frac{1}{2 \pi} \int_{0}^{2 \pi} \log \left|E\left(r e^{i \theta}\right)\right| d \theta-O(\log r) \\
& =(1+o(1))(\log r)^{2} .
\end{aligned}
$$

On the other hand, we have from the logarithmic derivative lemma that

$$
\frac{1}{2 \pi} \int_{0}^{2 \pi} \log \left|E^{\prime}\left(r e^{i \theta}\right)\right| d \theta \leq m\left(E^{\prime} / E, r\right)+m(E, r)=(1+o(1))(\log r)^{2} .
$$

Now (6) follows from (24) and (25). Thus Lemma 3 is proved completely.

Remark. Functions similar to those used here were considered in [14], [3], and [6]. Our functions are better behaved in the sense that inequality (23) holds for all large $r$ rather than for some large $r$.

Lemma 4. Let $E$ be the function in Lemma $3, m \geq 1$ be any integer, and $S(r)=\left\{z=\left(z_{1}, \ldots, z_{m}\right) \in \mathbb{C}^{m}:\|z\|=r\right\}$. Then

$$
\int_{S(r)} \log \left|E^{\prime}\left(z_{1}\right)\right| \sigma(z)=(1+o(1))(\log r)^{2} \quad(r \rightarrow \infty)
$$


and

$$
\int_{S(r)} \log \left|E\left(z_{1}\right)\right| \sigma(z)=(1+o(1))(\log r)^{2} \quad(r \rightarrow \infty) .
$$

Proof. The case $m=1$ is already treated in Lemma 3. So we assume $m \geq 2$ in the following. By an orthogonal projection of $\mathbb{C}^{m}$ to $\mathbb{C}$ (e.g. see [7]) and Lemma 3,

$$
\begin{aligned}
\int_{S(r)} \log \left|E^{\prime}\left(z_{1}\right)\right| \sigma(z) & =\frac{m-1}{\pi} \int_{0}^{r} \int_{0}^{2 \pi} \frac{\left(r^{2}-t^{2}\right)^{m-2}}{r^{2 m-2}} t \log \left|E^{\prime}\left(t e^{i \theta}\right)\right| d \theta d t \\
& =2(1+o(1))(m-1) \int_{0}^{r} \frac{\left(r^{2}-t^{2}\right)^{m-2}}{r^{2 m-2}} t(\log t)^{2} d t \\
& =(1+o(1)) G_{m}(r) .
\end{aligned}
$$

We use induction to prove $G_{m}(r)=(1+o(1))(\log r)^{2}$ for all $m$. If $m=2$, it is straightforward to show this claim holds. Suppose the claim is true for $m-1$. Then, taking the derivative of $G_{m}$ with respect to $r$ gives that

$$
\frac{d}{d r}\left(r^{2 m-2} G_{m}(r)\right)=2(m-1) r^{2 m-3} G_{m-1}(r)=2(m-1)(1+o(1)) r^{2 m-3}(\log r)^{2} .
$$

Thus we get

$$
r^{2 m-2} G_{m}(r)-G_{m}(1)=r^{2 m-2}(1+o(1))(\log r)^{2} \quad(r \rightarrow \infty),
$$

and the claim is verified. Hence (26) follows. Similarly we can prove (27) by using Lemma 3. So Lemma 4 is proved completely.

\section{Proof OF THE THEOREMS}

Proof of Theorem 1. By using a refinement of Ahlfors' method (e.g. see [5], p. 95), we have that

$$
T_{f, \kappa}(r)+\sum T_{f, \rho_{j}}(r)-N_{f, D}(r)+N_{f, \operatorname{Ram}}(r) \leq \frac{n}{2} \log \int_{S(r)} \gamma_{\Lambda}^{1 / n} \sigma-\frac{1}{2} \log \gamma_{f}(0)+1 .
$$

Set $B(t)=\left\{z=\left(z_{1}, z_{2}, \ldots, z_{m}\right) \in \mathbb{C}^{m} ;\|z\|<t\right\}$ and

$$
F(r)=\int_{0}^{r} \frac{d t}{t^{2 m-1}} \int_{B(t)} \gamma_{\Lambda}^{1 / n} \Psi
$$

Then, by ([1], Lemma 9), there is a constant $B$ such that

$$
F(r) \leq B T_{f, \eta}^{1+k / n}(r)
$$

Applying Lemma 2 for a sufficiently small $\varepsilon$ so that $\varepsilon B \leq 1$ and the $\log (\varepsilon B)$ 
term dominates the constant term in (28), we obtain from (29) that

$$
\begin{aligned}
\frac{n}{2} \log & \int_{S(r)} \gamma_{\Lambda}^{1 / n} \sigma-\frac{1}{2} \log \gamma_{f}(0)+1 \\
& =\frac{n}{2}\left(\frac{(m-1) !}{2 r^{2 m-1}}\left(r^{2 m-1} \frac{d F}{d r}\right)^{\prime}\right)-\frac{1}{2} \log \gamma_{f}(0)+1 \\
& \leq \frac{n}{2} \log \left(\varepsilon F(r) \psi^{2}\left(\varepsilon F^{1 / 2}(r)\right) \psi(r) / \phi^{2}(r)\right)-\frac{1}{2} \log \gamma_{f}(0)+1 \\
& \leq \frac{n}{2} \log \left(\varepsilon B T_{f, \eta}^{1+k / n}(r) \psi^{2}\left(\varepsilon B T_{f, \eta}^{(1+k / n) / 2}(r)\right) \psi(r) / \phi^{2}(r)\right)-\frac{1}{2} \log \gamma_{f}(0)+1 \\
& \leq \frac{n+k}{2} \log T_{f, \eta}(r)+n \log \psi\left(T_{f, \eta}(r)\right)+\frac{n}{2} \log \psi(r)-n \log \phi(r)
\end{aligned}
$$

for all $r \geq r_{0}$ outside a set $E$ with $\int_{E} d r / \phi(r)<\infty$. It follows that Theorem is proved.

Proof of Theorem 2. Let $z=\left(z_{1}, z_{2}, \ldots, z_{m}\right) \in \mathbb{C}^{m}$ and define

$$
f(z)=f\left(z_{1}, z_{2}, \ldots, z_{m}\right)=\left(1, f_{1}\left(z_{1}\right), f_{2}\left(z_{2}\right), \ldots, f_{n}\left(z_{n}\right)\right),
$$

where $f_{i}(z)=\exp \left(E\left(z_{i}\right)\right)$ for $i=1,2, \ldots, n$ and recall $m \geq n$. Clearly $f$ is a holomorphic mapping from $\mathbb{C}^{m}$ to $\mathbb{P}^{n}$. Let $L$ be the hyperplane bundle and $\eta$ be the Fubini-Study form $\omega_{0}$. Set $D_{i}=\left\{w_{i}=0\right\} \quad(i=0,1, \ldots, n)$. Then we take a divisor $D=\sum_{j=1}^{g} D_{j} \subset|L|$ and holomorphic sections $s_{j}$ of $L$ for which the corresponding $D_{j}$ are linear functions in the local coordinates $s_{\alpha j}=w_{j} / w_{\alpha}$ in $U_{\alpha}=\left\{w_{\alpha} \neq 0\right\}$, where $\left\{U_{\alpha}\right\}_{0}^{n}$ is a covering of $\mathbb{P}^{n}$. Thus the Hermitian moduli of the sections $s_{\alpha j}$ are

$$
\left|s_{\alpha j}\right|=\frac{\left|w_{j}\right|}{\left(\sum_{j=0}^{n}\left|w_{j}\right|^{2}\right)^{1 / 2}} .
$$

Therefore

$$
\begin{gathered}
\left|s_{j} \circ f\right|=\left|\exp \left(E\left(z_{j}\right)\right)\right| /|f|(j=1, \ldots, n), \quad\left|s_{0} \circ f\right|=1 /|f|, \\
|\Delta|=\left|\prod_{j=1}^{n} E^{\prime}\left(z_{j}\right) \exp \left(E\left(z_{j}\right)\right)\right|, \quad h_{k}=n ! /|f|^{2(n+1)} .
\end{gathered}
$$

It follows that

$$
\gamma(z)=\frac{|\Delta|^{2} h_{k}}{\prod_{j=0}^{n}\left|s_{j} \circ f\right|^{2}}=C \prod_{j=1}^{n}\left|E^{\prime}\left(z_{j}\right)\right|^{2} .
$$

Hence we have from ([5], p. 95) and Lemma 4 that

$$
\begin{aligned}
T_{f, \kappa}(r) & +\sum T_{f, \rho_{j}}(r)-N_{f, D}(r)+N_{f, \operatorname{Ram}}(r) \\
= & \int_{S(r)} \log \gamma^{1 / 2}(z) \sigma(z)+O(1) \\
= & n \int_{S(r)} \log \left|E^{\prime}\left(z_{1}\right)\right| \sigma(z)+O(1)=n(1+o(1))(\log r)^{2} .
\end{aligned}
$$


Since the Fubini-Study form coincides with the Chern form $c_{1}(L)$,

$$
\begin{aligned}
T_{f, \eta}(r) & =T_{f, \rho_{j}}(r)=\int_{0}^{r} \frac{d t}{t} \int_{B(t)} f^{*}\left(c_{1}(L)\right) \wedge \omega^{m-1} \\
& =\int_{0}^{r} \frac{d t}{t} \int_{B(t)} d d^{c} \log \|f\|^{2} \wedge \omega^{m-1}=\int_{S(r)} \log \|f\| \sigma .
\end{aligned}
$$

It follows from (31), (13), and (9) that

$$
\begin{aligned}
T_{f, \eta}(r) & =\int_{S(r)} \log \|f\| \sigma(z) \leq n \int_{S(r)}\left|E\left(z_{1}\right)\right| \sigma(z) \\
& \leq n \max _{\left|z_{1}\right|=r}\left|E\left(z_{1}\right)\right| \leq n \exp \left((1+o(1))(\log r)^{2}\right) .
\end{aligned}
$$

\section{Consequently}

$$
\log T_{f, \eta}(r) \leq(1+o(1))(\log r)^{2} \quad(r \rightarrow \infty) .
$$

In the other direction, we obtain from (31), Jensen's inequality, and Lemma 4 that

$$
\begin{aligned}
\log T_{f, \eta}(r) & =\log \int_{S(r)} \log \|f\| \sigma(z)+O(1) \geq \int_{S(r)} \log \log \|f\| \sigma(z)+O(1) \\
& \geq \int_{S(r)} \log \left|E\left(z_{1}\right)\right| \sigma(z)+O(1)=(1+o(1))(\log r)^{2} \quad(r \rightarrow \infty) .
\end{aligned}
$$

Combining (30), (32), and (33), Theorem 2 is proved.

\section{ACKNOWLEDGMENT}

The author thanks Min Ru, Linda Sons and the referee for helpful comments.

\section{REFERENCES}

1. W. Cherry, The Nevanlinna error term for coverings generically surjective case, Proceedings Symposium on Value Distribution Theory in Several Complex Variables (W. Stoll, ed.), Univ. of Notre Dame Press, 1993, pp. 37-54.

2. P. Hall, Nevanlinna error terms of some meromorphic functions, preprint.

3. A. Hinkkanen, $A$ sharp form of Nevanlinna's second fundamental theorem, Invent. Math. 108 (1992), 549-574.

4. S. Lang, The error term in Nevanlinna theory, Duke Math. J. 56 (1988), 193-218.

5. S. Lang and W. Cherry, Topics on Nevanlinna theory, Lecture Notes in Math., vol. 1433, Springer-Verlag, Berlin and New York, 1990.

6. J. Miles, $A$ sharp form of the lemma on the logarithmic derivative, J. London Math. Soc. 45 (1992), 243-254.

7. W. Rudin, Function theory in the unit ball of $\mathbb{C}^{n}$, Springer-Verlag, Berlin and New York, 1980.

8. L. R. Sons and Z. Ye, The best error terms of classical functions (to appear).

9. P. Vojta, Diophantine approximations and value distribution theory, Lecture Notes in Math., vol. 1239, Springer-Verlag, Berlin and New York, 1987.

10. P. M. Wong, On the second main theorem of Nevanlinna theory, Amer. J. Math. 111 (1989), 549-583.

11. P. M. Wong and W. Stoll, Second main theorem of Nevanlinna theory for nonequidimensional meromorphic maps, Amer. J. Math. 116 (1994), 1031-1071. 
12. Z. Ye, On Nevanlinna's secondary deficiency (to appear).

13. __, On Nevanlinna's second main theorem in projective space, preprint.

14. ___ On Nevanlinna's error terms, Duke Math. J. 64 (1991), 243-260.

Department of Mathematical Sciences, Northern Illnois University, DeKalb, IlliNOIs 60115

E-mail address: yecmath.niu.edu 